\title{
What buoyancy really is. A generalized Archimedes' principle for sedimentation and ultracentrifugation
}

Soft Matter 8, 7112 (2012)

Roberto Piazza, Stefano Buzzaccaro, Eleonora Secchi and Alberto Parola

Recommended with a commentary by M. Cristina Marchetti, Syracuse University

As an Italian physicist working at Syracuse University, in Syracuse, New York, a city that takes its name from the 2,700-year-old city of Siracusa, Italy, birthplace of Archimedes, I could not help but notice the recent article by Roberto Piazza and collaborators that generalizes Archimedes' principle, arguably one of the oldest and best known laws of physics. Archimedes' principle, formulated almost 23 centuries ago, states that a body immersed in a fluid experiences an upward thrust (or buoyant force) equal to the weight of the fluid it displaces [1]. In other words, objects heavier than water sink and objects lighter than water float. Reading a newspaper while floating effortlessly in the Dead Sea is an experience that really brings Archimedes' principle home. The law works very well at large scales, and it is often used to determine the density of bodies, although more accurate direct methods are also available.

It has been known for sometime, however, that discrepancies exist for particles or molecules suspended in complex fluids composed of many species of different size and density, when it is not obvious which density should be used in calculating the weight of Archimedes' "displaced fluid". For instance, in centrifugation experiments used to separate proteins or large biomolecules of different size by creating a controlled density gradient in the solvent it was found that the apparent density of the proteins inferred from conventional laws of buoyancy depends on the medium used to establish the density gradient [2]. Roberto Piazza and collaborators found a related curious effect when they added gold nanoparticles (which are 20 times denser than water) to an aqueous suspension of plastic particles just slightly denser than water and six times larger than the nanoparticles. The heavy gold nanoparticles floated to the top, forming a thin layer on the surface after a few days. This puzzling observation led them to rethink the very concept of buoyancy and formulate a generalization of Archimedes' principle that describes the behavior of colloidal particles immersed not in a simple fluid, but rather in a structured fluid or a colloidal suspension. In essence they demonstrated that in this case the amount of "displaced fluid" that enters Archimedes' law is modified by the density perturbation induced by the suspended particles on the surrounding complex solvent. In the specific example considered in the paper, the authors describe a binary colloidal mixture consisting of a very dilute density of type- 1 particles and a larger density of type- 2 particles in a solvent. The presence of particle 1 changes the distribution of particles 2 in a region surrounding particle 1 of size controlled by the range of the mutual radial correlation function $g_{12}(r)$. The density perturbation is largest in a spherical shell surrounding particle 1 of thickness given by the radius of particle 2 , where particles 2 are depleted due to 
repulsive interactions with particle 1 . The resulting deviation from uniform density in this region gives rise to an additional effective buoyant force on particle 1 given by

$$
F_{1}=-m_{2} g n_{2} \int\left[g_{12}(r)-1\right] d \vec{r}
$$

where $n_{2}$ is the number density of particle 2 and $m_{2}$ their buoyant mass. The pair correlation function $g_{12}(r)$ describes the variations in density surrounding particle 1 due to its interaction with particles 2 , which are oscillatory in nature, reflecting variations in packing. The buoyant force $F_{1}$ should be added to the usual Archimedes buoyant force $F_{0}=-\rho g V_{1}$, where $\rho$ is the mass density of the solvent and $V_{1}$ the volume of particle 1 . The authors further point out that in the presence of strongly attractive interactions between particles 1 and 2 the effect can be reversed, with particle 1 being pulled down by its surroundings, i.e. displaying an effective increased density.

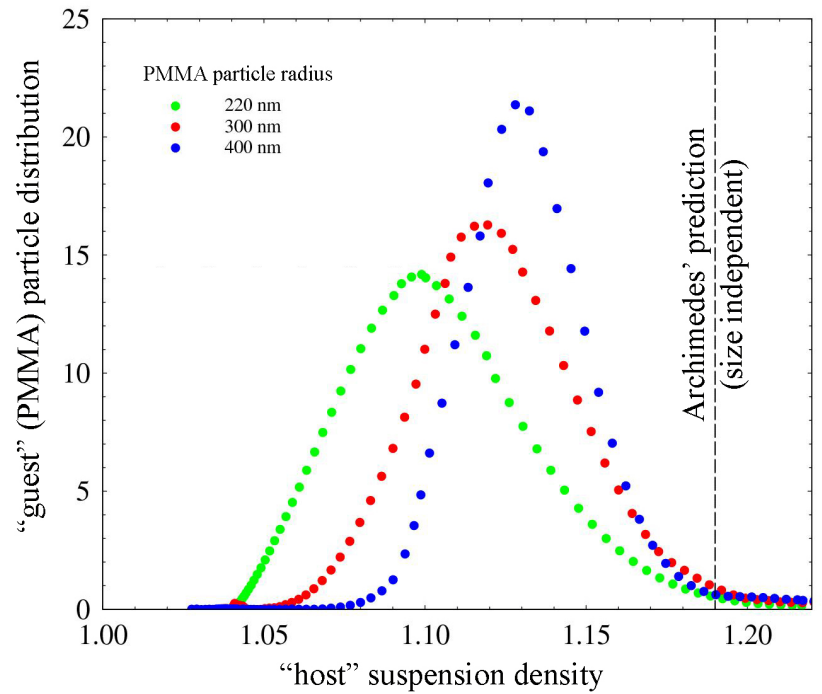

Fig. 1 - The density profiles of "guest" PMMA particles of three different sizes sedimented in the colloidal suspension described in the text as a function of the density of the "host" suspension. The vertical dashed line shows the size-independent value obtained using Archimedes' law. (Courtesy of Roberto Piazza)
Piazza and collaborators have tested their ideas by measuring the sedimentation profile of a moderately concentrated suspension of spherical and monodispersed nanoparticles in a solvent containing a dilute concentration of "guest" PMMA particles of three different sizes. The observed profiles of PMMA particles shown in Fig. 1 are not consistent with the simple law stated by Archimedes, but rather with the generalized form proposed by the authors. Even more striking is the fact that high-density nanoparticles added to the suspension will raise and accumulate in a layer above the lighter ones. Related effects have been seen in the sedimentation of other complex fluids, such as nematic liquid crystals in colloidal suspensions, where the sedimenting fluid forms complex phase stacks consisting of floating nematic layers sandwiched between isotropic regions of fluid [3].

Buoyancy and the associated phenomenon of sedimentation occur ubiquitously both in nature and in industrial processes. Sedimentation controls an enormous range of phenomena, from geological processes, such as the formation of certain rocks or the redistribution of pollutants created by human activities in river 
floodplains, to the shelf life of paints. When driven by centrifugation, it is used ubiquitously for blood analysis or to separate other biological macromolecules. The results of Piazza et al are important as they provide a simple, yet powerful tool for disentangling the effects of gravity and interparticle interactions in controlling the sedimentation of complex colloidal suspensions, which is at the hearth of all these phenomena.

[1] Archimedes of Syracuse, On Floating Bodies (287 BC- c. 212 BC).

[2] J. B. Ifft and J. Vinograd, The Buoyant Behavior of Bovine Serum Mercaptalbumin in Salt Solutions at Equilibrium in the Ultracentrifuge. II. Net Hydration, Ion Binding, and Solvated Molecular Weight in Various Salt Solutions, J. Phys. Chem. 70, 2814 (1966).

[3] D. de las Heras, N. Doshi, T. Cosgrove, J. Phipps, D. I. Gittins, J. S. van Duijneveldt $\&$ M. Schmidt, Floating nematic phase in colloidal platelet-sphere mixtures, Nature Scientific Reports 2, 789 (2012). 